Document downloaded from:

http://hdl.handle.net/10251/60185

This paper must be cited as:

Pérez Aparicio, JL.; Bravo, R.; Gómez-Hernández, JJ. (2014). Optimal numerical design of bucket elevators using discontinuous deformation analysis. Granular Matter. 16(4):485-498. doi:10.1007/s10035-014-0485-5.

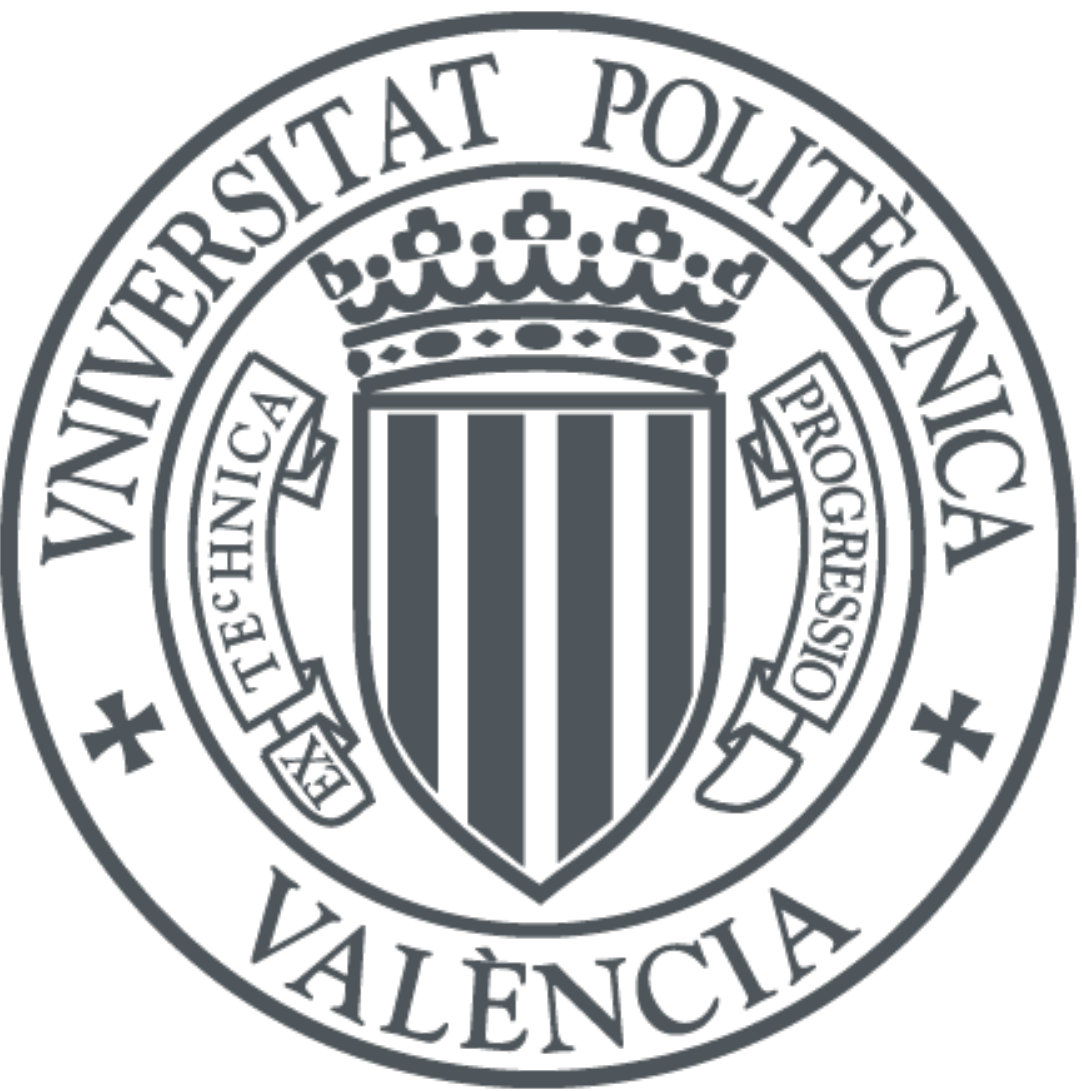

The final publication is available at

http://dx.doi.org/10.1007/s10035-014-0485-5

Copyright Springer Verlag (Germany)

Additional Information 


\title{
Optimal numerical design of bucket elevators using discontinuous deformation analysis
}

\author{
J.L. Pérez-Aparicio · R. Bravo · J.J. Gómez-Hernández
}

Received: date / Accepted: date

\begin{abstract}
Bucket elevators are efficient machines to transport granular materials in industrial and civil engineering applications. These materials are composed of hundreds, thousands or even more particles, the global behavior of which is defined by contact interactions. The first attempts to analyze the transportation of granular materials were treated by very simple continuum methods that do not take into account these interactions, producing simulations that do not fit the experimental results accurately. Given the internal discontinuity nature of granular media, it is reasonable to use numerical methods to model their behavior, such as Discontinuous Deformation Analysis (DDA) - a member of the Discrete Element Method family that started to be used in the 90's to analyze similar problems.

The version of DDA used in the current work treats grains as rigid circular particles with friction, damping and eventually cohesion with the objective of simulating and analyzing in detail the discharge of granular materials with bucket elevators. A deterministic com-
\end{abstract}

MFOM I+D (2004/38), MICIIN \#BIA-2008-00522 and Polytechnic University of Valencia grant PAID 05-10-2674.

J.L. Pérez-Aparicio

Department of Continuum Mechanics and Theory of Structures, Universitat Politècnica de València, 46022 Valencia, Spain

Tel.: +34 963877000 (Ext. 71815)

Fax: +123-45-678910

E-mail: jopeap@upvnet.upv.es

R. Bravo

Department of Structural Mechanics and Hydraulic Engineering, University of Granada 18071, Granada, Spain

E-mail:rbravo@ugr.es

J.J. Gómez-Hernández

Research Institute of Water and Environmental Engineering, Universitat Politècnica de València, 46022 Valencia, Spain

E-mail: jaime@dihma.upv.es puter code has been implemented and validated against simplified analytical formulae and experimental results taken from the literature. This computer code is then used to obtain optimum two-dimensional bucket geometries under specific working conditions. The optimization aims to maximize transport distance and to minimize remaining material, taking into account bucket velocity and the properties of the grains. The resulting geometries are discussed and compared against standard designs.

Keywords Discontinuous Deformation Analysis · Bucket elevators discharge - Numerical contact . Penalty method · Golden section algorithm · Bezier curves.

\section{Introduction}

Bucket elevators are machines that allow the continuous transportation of granular materials to a specific location under given conditions. They consist of a variable number of buckets attached to a moving belt or chain that transmits the motion to the buckets, see [27], [16] for a complete mechanical description. Although the functioning of the machine might seem simple, the study of the granular material movement requires the use of sophisticated numerical methods.

Water has been transported by bucket elevators since ancient times. The first modern bucket designs were used for transporting and discharging granular materials at low velocities (gravity discharge). In this situation, gravity is the responsible for the discharge and the material is simply poured into a nearby pile, with strong limitations in the amount and in the distance of the transported material. The experiments evidenced that the behavior of the grains was similar to that of 
a viscous fluid flow, therefore the shape of the bucket was simple and aimed to transport the maximum material volume. The need to increase volume and distance forced the rising of the operational velocity and, therefore, the development of discharge by the action of a centrifugal force. In this case, the mechanics of discharge is not simple anymore and requires a precise tool to analyze the behavior of the material inside the bucket.

Traditionally, the analysis of the discharge and design of buckets has been treated by analytical and experimental procedures based on practical but not fully realistic considerations; see [15] and [19] for states of the art of bucket elevator technology. The first works [16], [17] formulated, and contrasted with experiments, the trajectory of the center of gravity $(\mathrm{cg})$ of the granular material inside the bucket for centrifugal discharge. Later, addressing the problem of completely emptying by centrifugal discharge resulted in the experimental design of the "T-type" bucket [18]. Work continued with the development of a more detailed analytical formulation to describe the centrifugal and gravitational discharge resulting in an improvement of the operational efficiency under certain conditions [12], [13]. Although the latter formulation was based on complex mechanics, more specifically on kinematical considerations, it was only able to describe the evolution of the $c g$ and not of the whole system of particles.

Recent numerical techniques allow the analysis of the large number of grains that constitutes the granular flow inside the bucket. In section 2, we introduce a method that simulates the micromechanics of the grains called Discontinuous Deformation Analysis (DDA), a displacement-based method similar (in the contact mechanics sense) to the force-based Discrete Element Method (DEM) (see e.g. [29] and [25]). The DDA method is particularly attractive for modeling granular material problems with internal discontinuous geometry. Its applicability ranges from large scale problems (i.e., the mechanical response of rock assemblies and masonry, [28], [24], [26]) to small scale ones (i.e., a previous work for granular discharge of bucket elevators in [25]). The newer reference [22] completes [25]. DDA considers the global behavior of the particles through the analysis of the individual behavior of each member plus the interaction of particles by friction and non-penetrating contacts, resulting in a single momentum balance formulation with the addition of contact restrictions.

Some examples on the application of DEM to industrial problems can be found in [20] for a convey filled with wood pellets, and in [31] for the analysis of powder behavior in a rotatory drum. Additionally, DEM has been used to optimize several other discontinuous problems: Mixtures of powders [8], efficient shape for soil-tillage interaction [30], rock cutting conditions for tunnel boring machines [23] and wear reduction in ball mills [21]. In the previous references, the election of the optimal parameters is based on brute force analyses of a very large number of cases with different parameter values. Optimization algorithms are not used and therefore, a high computational and post-processing cost is needed. This cost can be reduced as described in [1], which analyzes and optimizes the discharge time and flow of a hopper using multiobjective optimization procedures.

This article develops and validates the contact DDA method necessary for the complete analysis of bucket elevators. In addition, the "gradual deformation optimization algorithm" [9] is used to calculate the shape that provides the optimum discharge of a given granular material under given working conditions. The resulting computer code allows to identify the most significant aspects of the bucket geometry regarding the granular discharge, to analyze the dynamics of the granular flow and to design the optimal shape of the bucket. Section 3 describes the discharge process from the granular flow point of view and lists three types of generic, commonly used, bucket types. In section 4, the numerical aspects of the procedure are developed; first, the trajectory of a single particle inside a bucket is simulated and compared existing analytical formulae; second, a similar comparison is done for discharges of a real granular mass, the experimental results of which are taken from the literature. In section 5, simulations of gravitational and centrifugal discharges using thousands of particles are presented, which serve to identify the granular material flow. In order to simulate compaction during filling, an orderer array of particles is dropped into the bucket from a certain height under the action of gravity. A parametric analysis of the discharge as a function of rotation speed and a comparison with experimental results are presented in section 6 . Section 7 ends the article with the optimization of the bucket shape for several operational conditions and materials, and for several bucket types.

\section{Discontinuous Deformation Analysis}

In two-dimensional modelling, granular materials are represented as an aggregate of polygonal particles with highly variable sizes. Polygons can be replaced with disks or with other complex forms easy to paremeterize, such as ellipsoids. DDA is able to analyze the physics of discontinuous media using rigid disks and frictional contacts at a reduced computational cost. This analysis includes the simulation of particle interactions among 
themselves and with the containers, i.e., with the physical boundaries.

\subsection{Formulation of DDA}

DDA is based on the laws of classical mechanics, more specifically on Hamilton's Principle, an energy method constructed with the Lagrange function:

$\mathscr{L}(U, \dot{U})=\sum_{i=1}^{n}\left[\mathscr{T}\left(\dot{U}^{i}\right)-\mathscr{V}\left(U^{i}\right)\right]$

where $\mathscr{T}\left(\dot{U}^{i}\right), \mathscr{V}\left(U^{i}\right)$ are the kinetic and potential energies of a generic body numbered $i=1, \ldots, n$. These energies depend on velocity $\dot{U}^{i}$ and displacement $U^{i}=$ $\left\{u^{i}, v^{i}\right\}$, functions defined $\forall x, y \in i$. Applying Hamilton's Variational Principle of Minimal Action, the corresponding Euler-Lagrange equation is:

$\frac{d}{d t} \frac{\partial \mathscr{T}}{\partial \dot{U}^{i}}-\frac{\partial \mathscr{V}}{\partial U^{i}}=0$

This equation leads to the differential equations of motion of a system composed of $n$ particles:

$M \ddot{U}+C \dot{U}+K U=F(U, t)$

In Eq. (3), $M, C$ and $K$ are the mass, damping and stiffness matrices of a nonlinear system subject to a load $F$ dependent on time and displacement. DDA calculates an approximation of $U^{i}$ at any point from the displacements $D^{i}(t)$ of a designated point by means of a linear combination of shape functions (Eq. 4). The designated point lies inside the body, usually it is its $c g$, and its variables carry subindex $o$. The simplest approximation of $U^{i}$ is obtained using first-order shape functions. Given that granular materials are often composed of rock fragments of high Young's modulus, the material can be considered nearly incompressible. Therefore, the kinematic hypothesis of movement of a rigid solid is the most appropriate:

$$
\underbrace{\left(\begin{array}{c}
u^{i} \\
v^{i}
\end{array}\right)}_{U^{i}(x, y, t)}=\underbrace{\left(\begin{array}{ccc}
1 & 0 & -\left(y-y_{o}^{i}\right) \\
0 & 1 & \left(x-x_{o}^{i}\right)
\end{array}\right)}_{\mathscr{S}^{i}(x, y)} \underbrace{\left(\begin{array}{c}
u_{o} \\
v_{o} \\
\gamma_{o}
\end{array}\right)}_{D^{i}(t)}
$$

where $u_{o}^{i}, v_{o}^{i}$ are the horizontal and vertical displacements of the $c g$ with coordinates $x_{o}, y_{o}$, and $\gamma_{o}$ the rotation of the body around this $c g$, the three of them grouped in $D^{i}$. Inserting Eq. (4) into the Euler-Lagrange Eq. (2) provides the discrete equations of motion:

$M \ddot{D}+C \dot{D}+K(D) D=F(D, t)$
These equations have to be integrated in time applying discrete methods such as the traditional family of Newmark- $\beta$ algorithms with initial conditions $D(0)=$ $D_{0}$ and $\dot{D}(0)=\dot{D}_{0}$.

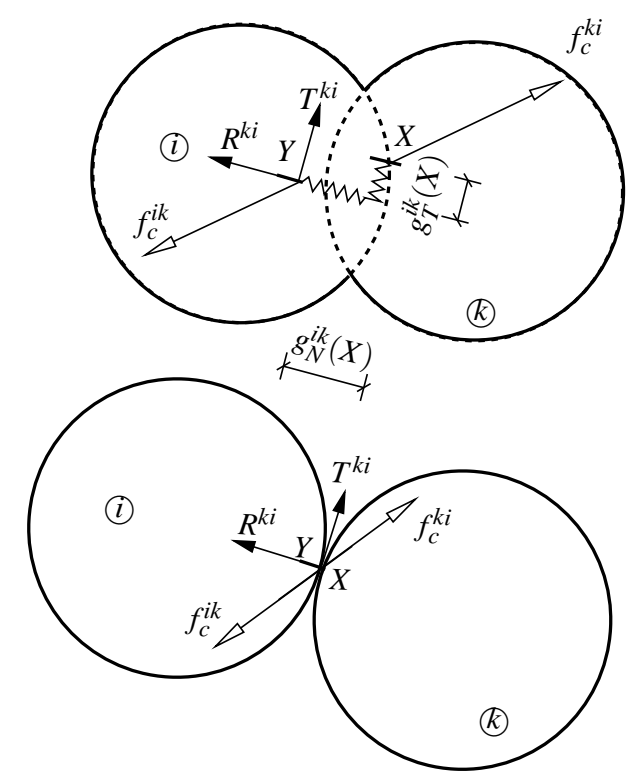

Fig. 1 Penalty method: Contact and penetration between two circular bodies, top. Distances $g_{N}^{i k}$ define maximum penetration, $g_{T}^{i k}$ tangential displacement. After convergence, $g_{N}^{i k} \approx$ $g_{T}^{i k} \approx 0$, bottom.

\subsection{Numerical contact}

DDA simulates the interaction among rigid particles through non-penetrating contacts and friction. Figure 1 top depicts the non-realistic situation of two interpenetrating bodies. The gap function $g_{N}^{i k}(X)$ is used to measure the penetration between the two particles and is used to impose non-penetration through the constraint:

$g_{N}^{i k}(X)=[X-Y] R^{i k} \geq 0$

The vectors $X, Y$ define the coordinates of the two closest points in bodies $i, k$, and $R^{i k}=-R^{k i}$ is the normal vector at the contact point. In reality, the bodies should be either in contact $g_{N}^{i k}=0$ or separated $g_{N}^{i k}>0$. Some small penetration $g_{N}^{i k}<0$ may result since the inequality Eq. (6) cannot be enforced exactly in the numerical computation.

To describe the motion in the tangential direction of the contact points, an additional kinematic condition introducing the tangential gap $g_{T}^{i k}$ is necessary:

$g_{T}^{i k}(X)=\left[X+u^{i}(X)-Y-u^{k}(Y)\right] T^{i k}$ 
where $u^{i}(X), u^{k}(Y)$ are the displacements related to a certain time increment of the contact points at both bodies, and $T^{i k}$ is the tangential unit vector also at the contact, Figure 1. To dynamically prescribe the nonpenetration, the method imposes a contact force:

$f_{c}^{i k}=f_{c N}^{i k} R^{i k}+f_{c T}^{i k} T^{i k}$

in which $f_{c N}^{i k}, f_{c T}^{i k}$ are the components of the contact force in the normal and tangential directions. For the case of two rigid rounded bodies, this force is applied at a single point. The tangential displacement is governed by the frictional Coulomb's law with a sliding function:

$\Phi^{i k}=f_{c T}^{i k}-\left|f_{c N}^{i k}\right| \mu \leq 0$

where $\left|f_{c N}^{i k}\right| \mu$ is the frictional force, $\mu=\tan \phi$ the friction coefficient and $\phi$ the friction angle. When $\Phi^{i k} \geq 0$ sliding starts and $g_{T}^{i k} \neq 0$; when $\Phi^{i k}<0$ rolling occurs and $g_{T}^{i k}=0$. Both situations are considered mutually exclusive.

The constitutive equation for the contact force is frequently modeled by penalization techniques, e.g. see [7] and references therein. The key idea is to introduce two parameters $K_{N}, K_{T}$ related to two high stiffness elastic springs placed between the contact points of the bodies along the normal and tangential directions (see Figure 1 bottom). The associated potential energies $\mathscr{V}$ are expressed as $K_{N} g_{N}^{i k} 2 / 2, K_{T} g_{T}^{i k 2} / 2$ for rolling, and $K_{N} g_{N}^{i k} 2 / 2,\left|f_{c N}^{i k}\right| \mu g_{T}^{i k}$ for sliding. Inserting these energies in Eq. (2) provides the contact forces needed in Eq. (8): $f_{c N}^{i k}=K_{N} g_{N}^{i k}$ and $f_{c T}^{i k}=K_{T} g_{T}^{i k}$ for rolling, $f_{c N}^{i k}=K_{N} g_{N}^{i k}$ and $f_{c T}^{i k}=\mu f_{c N}^{i k}$ for sliding.

The calculation of precise non-penetrating contacts is sensitive to a proper tuning of $K_{N}$ and $K_{T}$. Among several alternatives to model non-penetrating contacts [3], we adopt the penalization approach because it has been shown to be both accurate and efficient for problems with large number of contacts such as the ones studied in this work.

\section{Basic theory of discharge and types of buckets}

The bucket elevator is a machine to transport granular industrial materials in the vertical direction or along inclined planes. The design and performance of the elevators vary with the characteristics of the material, and must at least consider the geometry of the bucket and the operational speed.

Emptying the buckets can be done in two principal ways: By action of gravity or by centrifugal force (Figure 2 left and right, respectively). In the former, the material falls along the inner edge of the bucket and is thus guided to the discharge mouth or deflector, Figure 3. This type of discharge occurs for low operating speeds 0.5 to $0.8 \mathrm{~m} / \mathrm{s}$. Centrifugal discharge is performed by emptying the material along the outer wall by the action of centrifugal force, activated when the movement changes from linear to circular. Early

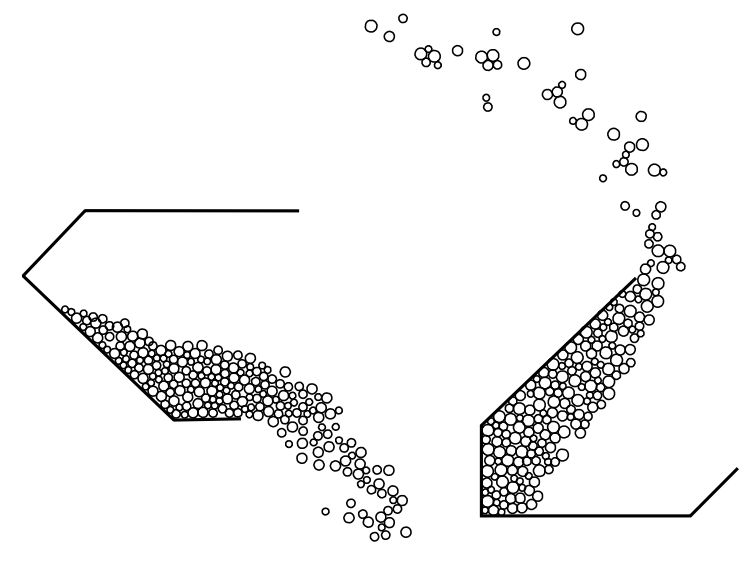

Fig. 2 Gravity (left) and centrifugal (right) discharges for a straight bucket during rotation.

studies of granular material discharge considered the material mass lumped at a single point located at the initial $c g$. The approach assumes that this $c g$ maintains a constant distance from the center of rotation $(c r)$ of the bucket. For gravity elevators this fact is generally correct, as Beckert and Foll [2] demonstrated in 1966. However, in the following numerical simulation section, we will show that the $c g$, in general, does not occupy a constant position but rather moves along and outside the outer wall of the bucket, as is the case of centrifugal discharge. Thus, the distance $c g-c r$ increases as a function of the bucket opening angle, and for a constant rotation speed the forces on the material decrease progressively.

In any case this simplification is useful for certain simple calculations, such as obtaining the important angle of detachment -angle at which the material leaves the bucket. The results of these calculations, complemented with many experiments, have resulted in the following criteria for bucket elevator design (Figure 3):

- It is advisable to use buckets designed with the deflector inclined about $45^{\circ}$.

- Deep, high-capacity buckets are defined by a tulipshaped outer wall with double curvature.

- The deeper the bucket and the smaller its opening angle, the harder will be to empty the contents, specially under high centrifugal action. 
- A shallow bucket must have a large exit perimeter and a variable opening angle.

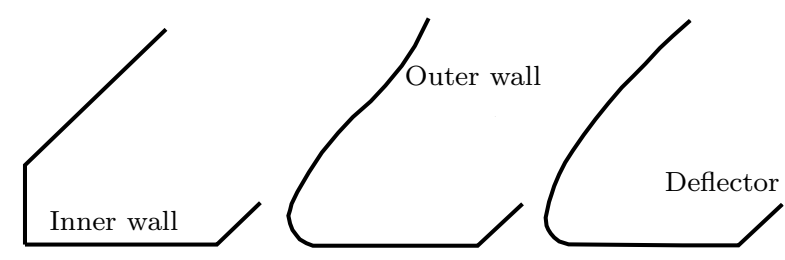

Fig. 3 Straight bucket, left. T-type, outer wall with double curvature, middle. Logarithmic, outer wall with logarithmic spiral, right.

The first item is mostly related with gravitational discharge, for which optimal and controlled residual emptying is obtained at all times, and large amounts of material can be transported although at short distances. The second item refers to the so called T-type, this design expels the material prematurely between predischarge and discharge phases. It can also achieve optimal emptying and is appropriate for all kinds of granular solids. The third item implies that to obtain good results in fast-moving installations the bucket must have a sufficiently large opening angle as in Figure 3 middle and right. The fourth item applies to the handling of fine-grained or dust-like materials, which have low cohesion and large fluidity. In this design, the outer wall adopts the form of a logarithmic spiral, which results in a progressive decrease of the opening towards the outer edge. This shape causes the material to compact in its way out, making discharge somewhat more difficult, but achieving better overall performance than the conventional design.

The best design should adapt the bucket geometry to the required transportation distance, the amount and properties of the material, and should aim at the complete emptying of the bucket. Section 7 shows optimal designs considering all of these objectives.

\section{Numerical validations}

This section studies the penalty, a critical parameter of the numerical model. It is necessary to tune this parameter to avoid unreal interpenetrations of the grains. The section also analyzes the performance and describes the evolution of representative granular materials inside the buckets during the filling and discharge phases.

\subsection{Optimal value of the penalty parameter}

Low values of the penalty parameter will result in large penetrations that must be avoided. A possible solution is to sequentially increase the parameter, for example by $25 \%$, when these interpenetrations exceed a certain tolerance.

Table 1 shows a sensitivity analysis to the choice of the initial value of the penalty parameter for the simulation of a discharge by gravity. The implementation of this discharge is described in detail in section 5 , and it assumes that $K_{N}=K_{T}$. It presents the initial value chosen, the final value at which the sequential approach described above arrives, and the number of iterations needed for the numerical model to converge. From the results, we can appreciate that for initial values of the penalty of $10^{6} \mathrm{~N} / \mathrm{m}$, the algorithm does not need to increase the penalty in any of the contact iterations for the code to converge. We thus find appropriate a value of $10^{6}$, or slightly larger, for these type of problems, with a significant reduction of iterations.

Table 1 Evolution of the penalty parameter $K_{N}=K_{T}$ in N/m and number of required iterations for a complete gravitational discharge simulation.

\begin{tabular}{ccc}
\hline Initial & Final & \# iterations \\
\hline $10^{3}$ & $2.3 \cdot 10^{8}$ & 4400 \\
$10^{4}$ & $4.1 \cdot 10^{7}$ & 2400 \\
$10^{5}$ & $7.4 \cdot 10^{6}$ & 1900 \\
$10^{6}$ & $1.0 \cdot 10^{6}$ & 900 \\
\hline
\end{tabular}

\subsection{Validations}

DDA results are validated in this section against an analytical formulation from [11] and [4]. These references provide the position vector $S$ Eq. (10) and the corresponding velocity and acceleration $\dot{S}, \ddot{S}$, for the discharge of a particle around a pulley with radius $r$ $=500 \mathrm{~mm}$.

$$
\begin{aligned}
S= & x e^{\lambda_{1} t}+y e^{\lambda_{2} t}-\mu r+\frac{g}{2 \omega^{2}\left(1+\mu^{2}\right)} . \\
& {\left[-\left(1-\mu^{2}\right) \sin \left(\alpha_{0}+\omega t\right)+2 \mu \cos \left(\alpha_{0}+\omega t\right)\right] }
\end{aligned}
$$

where $\lambda_{1}, \lambda_{2}=\omega\left(\mu \pm \sqrt{1+\mu^{2}}\right), g$ is the gravity acceleration, $\omega$ the rotational velocity, $t$ time and $\alpha_{0}$ the bucket position angle at $t=0$; see [5], [11] for definitions of straight and curved outer walls. This solution is limited, since it is applicable only to a single particle and does not take into account its rolling.

In the first two simulations (Figure 4), we compare the numerical and analytical results for gravity and centrifugal discharges. The material density, not considering voids, is $2500 \mathrm{~kg} / \mathrm{m}^{3}$, with particles of $2 \mathrm{~mm}$ 
diameter. The friction coefficient between particles and bucket surface is $10^{\circ}$ without cohesion. The initial value of the penalty parameter is chosen as $10^{6} \mathrm{~N} / \mathrm{m}$.

Figure 4 shows the numerical and analytical trajectories during rotation for gravity discharge, $\omega=2.4$ $\mathrm{rad} / \mathrm{s}$, and for centrifugal discharge, $\omega=8.9 \mathrm{rad} / \mathrm{s}$. The origin of coordinates $(0,0)$ is situated at the pulley's $c r$. For gravity discharge the correspondence is very good.

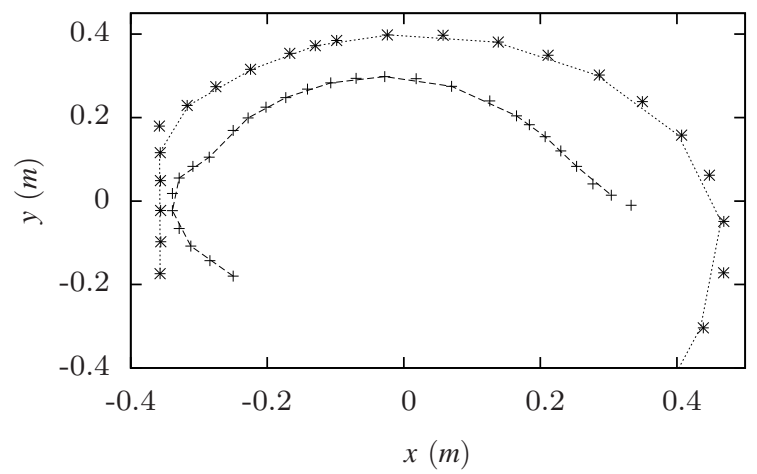

Fig. 4 Analytical (line) and numerical (symbol) trajectories of a single particle for centrifugal discharge and straight bucket (exterior graph). Idem for gravity discharge and any bucket (interior graph).

For centrifugal discharge the correspondence is again very good with an small exception at the beginning, the analytical formulation does not account for the sudden appearance of a small centrifugal acceleration, which causes the particle to rise and hit the outer wall. The slight variation of trajectories at the end of both discharges is due to a greater exit velocity predicted by DDA, since it accounts for particle rotation.

Next, in Figure 5 the numerical prediction of the centrifugal discharge for two particles located on the outer wall of a straight bucket is compared with the analytical prediction and the experimental results from [4]. One particle is located deep inside the bucket and the other one close to the edge. The differences are noticeable at the beginning of the rotation, as before, they are due to the sudden change from linear to circular displacement. This effect is not considered by the analytical formulation, although it is only important before the bucket reaches $\mathrm{a} \approx 40^{\circ}$ angle; after this, the trajectories resemble closely each other. Table 2 shows the rotation angles of initial discharge and initial sliding for the three trajectories. All results show considerable similarity; it is evident that the numerical and analytical models are able to approximate well the movement of a single particle. The only exception is the angle of sliding of the outer particle, for which the initial conditions in the experiment are uncertain.

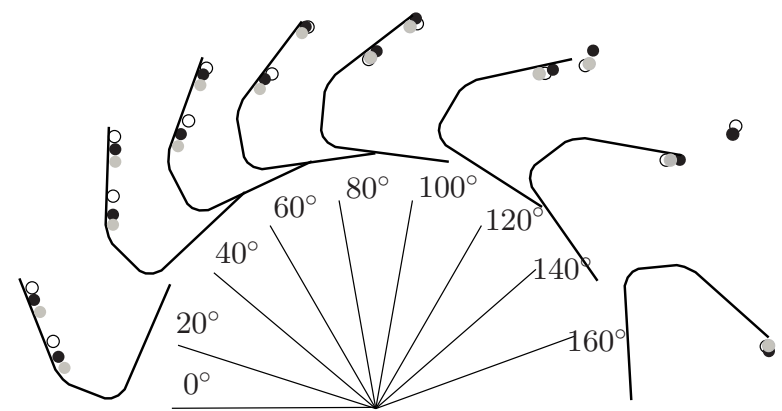

Fig. 5 Centrifugal discharge. Experimental (open circle), numerical DDA (black circle) and analytical (grey circle) results for two particles in different starting positions.

Table 2 Angles of sliding and discharge for two particles, one located deep into the bucket, and another one located close to the edge. Experimental, analytical and numerical results.

\begin{tabular}{lccc}
\hline & Exp. & Anl. & DDA \\
\hline Outer particle sliding & $50^{\circ}$ & $67^{\circ}$ & $62^{\circ}$ \\
Inner -"- & $86^{\circ}$ & $83^{\circ}$ & $84^{\circ}$ \\
Outer particle discharge & $113^{\circ}$ & $116^{\circ}$ & $114^{\circ}$ \\
Inner -"- & $162^{\circ}$ & $160^{\circ}$ & $155^{\circ}$ \\
\hline
\end{tabular}

Finally, Figures 6 represents the analytical results from the formulation by [11] and the numerical ones by DDA of the centrifugal trajectories followed by a particle inside a T-type and inside a logarithmic bucket. In both figures, every tick indicates the position of the particle spaced at constant time intervals. The results show that the T-type favor centrifugal discharge, as confirmed by the larger distance between ticks. Notice also that this design is able to force the material to reach a higher distance in a lower time than the logarithmic design as measured by the slope of the curve when $x>0.8 \mathrm{~m}$, and that this bucket expels the particle prematurely with higher velocity, as observed in the change of slopes at $x \approx 0.32$ for T-type and $x \approx 0.4$ for logarithmic. For discharge by gravity, all trajectories would coincide due to the equal geometry of the inner wall, see Figure 4.

\section{Numerical results}

In this section, several cases of realistic processes of discharge under common conditions in the transport of granular material industry are simulated by DDA showing its ability to model the behavior of granular materials.

To the best of our knowledge, there is no analytical formulation in the literature to describe problems with a large number of particles; therefore, the only means of validation are based on descriptions and in some cases experimental results obtained from [5], [14] and [4]. As 

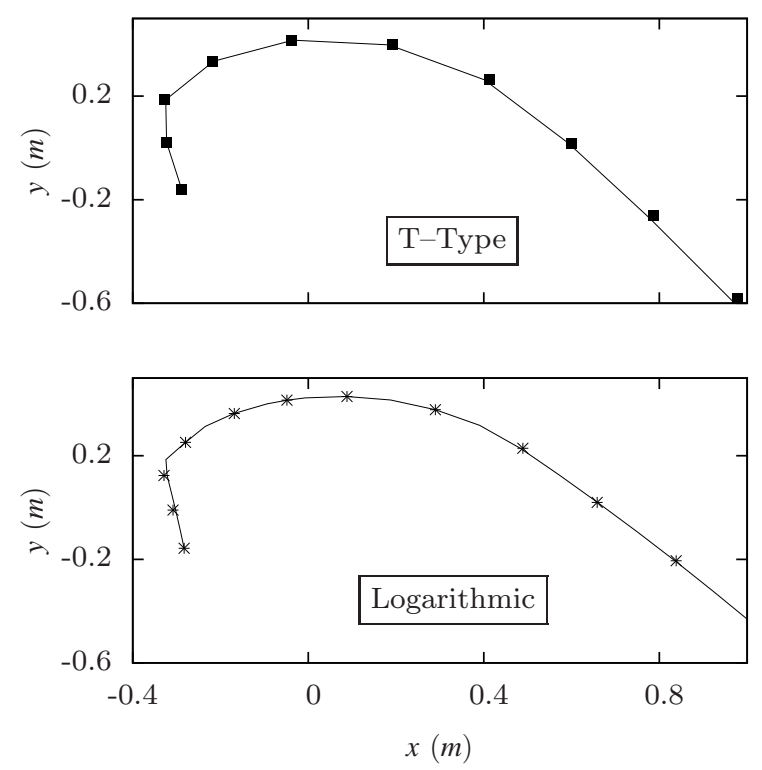

Fig. 6 Analytical (line) and numerical (symbol) trajectories of a single particle for centrifugal discharge for two bucket types.

in any process of transporting granular material, we will tackle the phases of filling, transport itself and discharge.

The penalty parameter used for the simulations of this section is now $10^{7} \mathrm{~N} / \mathrm{m}$, obtained by trial and error as explained in the previous section. The penalty has been increased with respect to the one obtained in section 4.1 because many discharges are centrifugal and the number of particles has been considerably increased, what implies that higher impact forces will appear requiring a higher penalty.

\subsection{Filling the buckets}

The filling of the bucket is a process by which the material adopts the form of the container that holds it. This moulding process is facilitated by the semifluid characteristics of the particles, which reach a stable configuration through the process of minimizing the energy of the whole. During the process, all particles are in contact with the surrounding ones or with the walls of the bucket, see Figure 7. In reality, the buckets are filled in multiple ways, but the numerical simulation assumes that the discharge is by vertical loading of particles with diameters between 3.5 and $4.5 \mathrm{~mm}$, original disposed in a compact rectangular array.

The sample of about 700 particles has a solid volume of $252 \mathrm{~cm}^{3}$, which nearly fills the bucket to the top. We have adopted an angle of friction within the material of $47^{\circ}$ and between material and bucket of $34^{\circ}$.
With these parameters, the resulting density (including voids) is $600 \mathrm{~kg} / \mathrm{m}^{3}$. The properties do not correspond to real granular materials but to plastic beads, a convenient material for repetitive experiments used by [4]. The different grey levels in Figure 7 allow the visualization of the particle redistribution through the filling process.

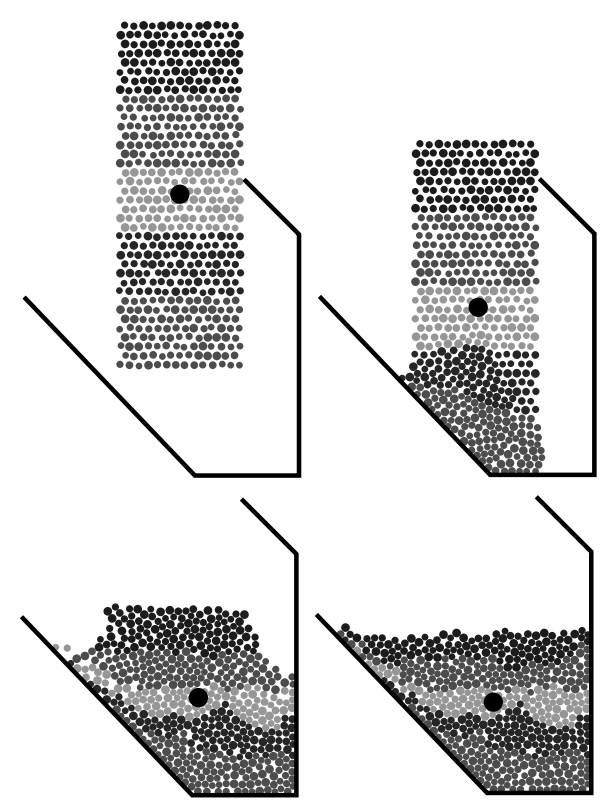

Fig. 7 Filling a straight bucket. Large bullet represents the material center of gravity, gray shades associated to the initial horizontal strata.

Starting from a statically ordered and separated matrix of the sample, the filling progresses under gravity. Since the particles are dropped from a relatively high position, the final free surface of the material is approximately flat, corresponding to a stable equilibrium (minimum energy). Note that after the filling, the layers are almost parallel and moulded to the shape of the bucket. The position of the $c g$ is represented by a thick bullet. Fillings for the three buckets (Figure 3) have been simulated, although for brevity only one is shown.

\subsection{Gravity discharge}

The filled bucket is subject to a rotation around a pulley of radius $0.5 \mathrm{~m}$ with an initial and constant velocity of $1.68 \mathrm{rad} / \mathrm{s}$. In this simulation, we use the mechanical characteristics and material properties established in the previous subsection. Figure 8 shows the positions of the bucket, the material distribution and its $c g$ for rotation angles in increments of approximately $20^{\circ}$. Unfortunately, we have not found experimental results to compare with the numerical ones; we can only 


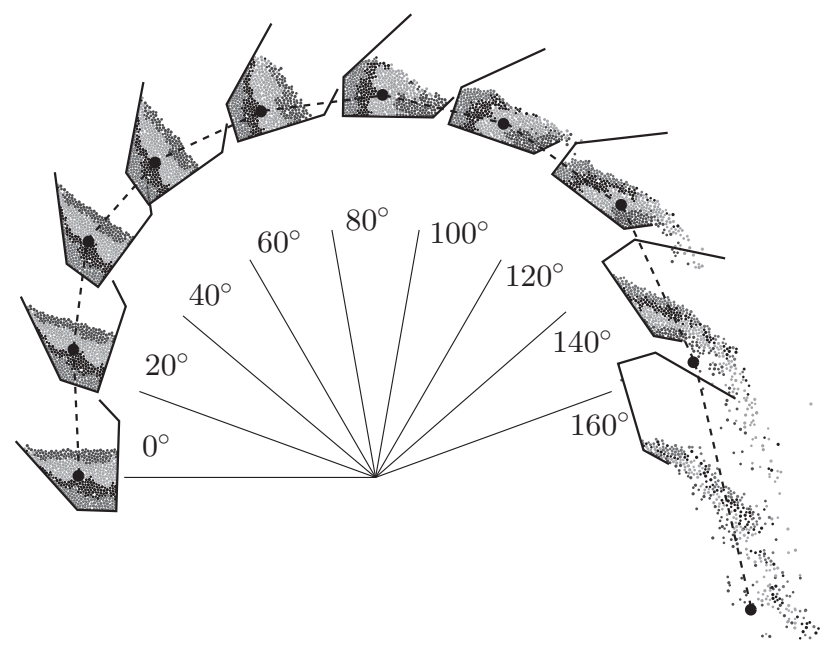

Fig. 8 Simulation of gravity discharge from a straight bucket; trajectory of the system's gravity center. Strata indicated by gray shades.

use the explanations and descriptions (not full experiments) given in [5]. From the analysis of Figure 8 we reach the following technological conclusions:

- When rotation starts, there is no displacement towards the bucket's exterior since weight is the dominant force.

- At $70^{\circ}$, the particles' movement is small due to weight and friction, but after $90^{\circ}$ the weight, helped by the centrifugal force, induces movement and provokes the discharge.

- The $c g$ position does not change before $90^{\circ}$; after that, it approaches the deflector and finally moves outside the bucket.

- The outer layers slide more easily than the deepest ones, since for the latter, friction is large due to the large normal component induced by the contacts. The particles close to the inner wall only move during the final phases.

- Discharge starts after the bucket passes the highest point, finalizing only when rotation is complete.

- Layers that initially are at the bottom turn to the top at the end. This effect forces all layers to be discharged as a block; in particular, particles initially in contact with the outer wall are discharged first.

As mentioned, the results from Figure 8 strongly depend on the penalty parameter; different values can give very different responses, some of them non realistic. Figure 9 shows the percentage of material remaining inside a straight bucket as a function of the angle of rotation for different values of the penalty. The figure indicates that very low values $\left(<K_{N}=1.5 \cdot 10^{5} \mathrm{~N} / \mathrm{m}\right)$ give poor results, predicting an unrealistic "explosion" at the beginning of the rotation. For intermediate values $<6 \cdot 10^{5}$ this effect is also present at the beginning,

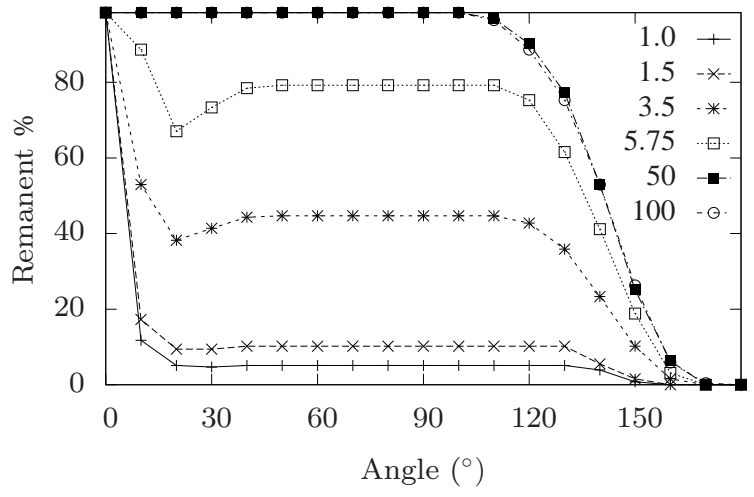

Fig. 9 Simulated remaining material in \% vs. rotation angle for a straight bucket and gravity discharge, for different penalty parameter values $\left(K_{N}=\right.$ number in legend $\left.\times 10^{5} \mathrm{~N} / \mathrm{m}\right)$.

but, after $\approx 30^{\circ}$, a more realistic holding of the material is predicted up to $110^{\circ}$, with a smooth discharge after. Note that the change of curve slope denotes recapturing of material, a negative phenomenon that sometimes occurs in real discharges although probably not in this case. Finally for $K_{N} \geq 50 \cdot 10^{5}$ the simulation is completely realistic and convergent: The smooth discharge starts only after $110^{\circ}$, finishing almost at the end of the rotation. The upper limit for $K_{N}$ is set by instabilities in the numerical simulator due to the poor conditioning of some matrices that must be inverted.

A physical explanation for the variation of results with the penalty parameter comes from the fictitious energy that is introduced by the fictitious springs, see reference [6]. A simplified expression of this energy is $E=K_{N} g_{N}^{2} / 2$. Since the lower the penalty, the larger the penetration allowed, the total energy will grow unbounded for diminishing values of the penalty; whereas, for very large values of the penalty, the penetrations are very small, and the energy will grow with the penalty parameters. The optimal penalty value will fall in the middle. The smaller the penalty $K_{N}$, the larger the quadratic penetration and consequently the added energy, resulting in unbounded displacements.

\subsection{Centrifugal discharge}

The structure of this subsection is almost the same as that of the previous one, although for centrifugal discharge the rotation speed is increased up to $8.9 \mathrm{rad} / \mathrm{s}$.

Experimental results are available for a logarithmic bucket from [4] allowing us to compare them with the numerical ones. Starting from the same filling process of subsection 5.1, calculations are performed for straight, T-type and logarithmic buckets. For the sake of brevity, 


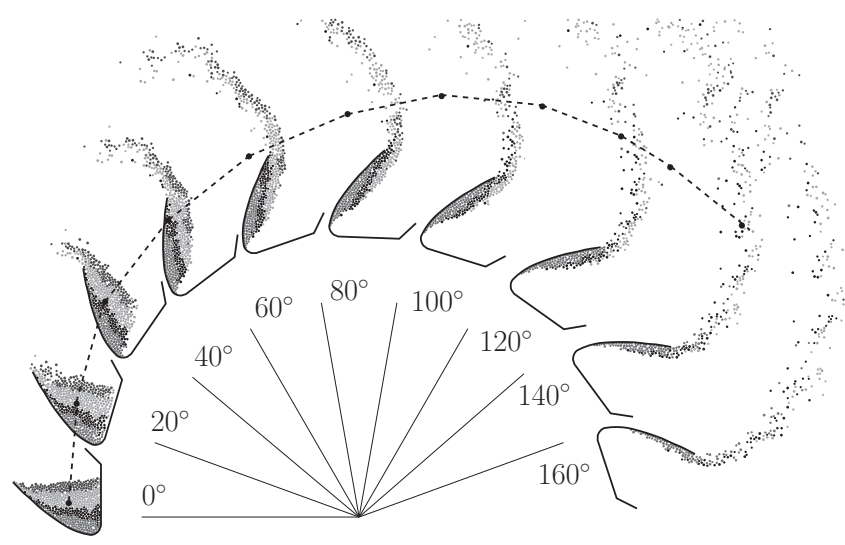

Fig. 10 Simulation of centrifugal discharge from a logarithmic bucket; trajectory of the system's gravity center. Strata indicated by gray shades.

we describe next only the results for the last type. These numerical results (Figure 10) confirm the results of the laboratory experiment:

- The movement starts at the inner wall: Particles move outwards in the direction of the centrifugal force, and a slight convexity appears in the free surface.

- At $\approx 40^{\circ}$ particles exit from the outer wall, discharge begins.

- Afterwards, the layers initially at the bottom slide along the outer wall and start exiting at $90^{\circ}$.

- Through the discharge phase, no significant distortion occurs in the layers.

- During most of the discharge the particles are located in the outer wall, with the inertial forces moving them forward.

- Part of the expelled material lags behind the bucket but eventually is discharged ahead of it.

- The $c g$ moves away from the bucket through the discharge process.

The last item invalidates the hypothesis of a fixed location of the $c g$ within the bucket, assumed in many analytical studies.

For the second centrifugal simulation, experimental data from a controlled experiment [4] and numerical results are compared, Figure 11. The sample is composed of plastic spheres of $3 \mathrm{~mm}$ diameter, with the density and friction given in subsection 5.1. In the experiment, the spheres are deposited manually with compact ordering, an initial condition that we can replicate exactly in our numerical simulations. A straight bucket (M-type, according to [4]) is subject to $8.58 \mathrm{rad} / \mathrm{s}$ on a pulley of diameter $0.5 \mathrm{~m}$.

DDA results are clearly inside the experimental ones, although we observe that DDA predicts a slightly more forward away trajectory than the experiments. This is

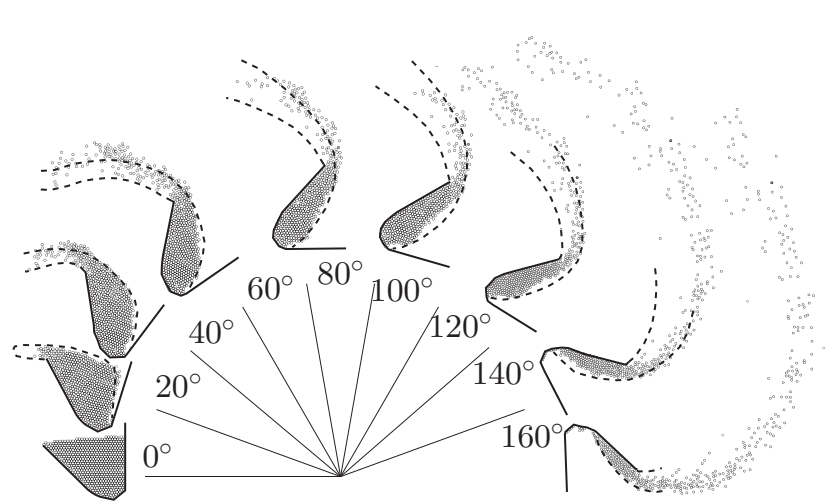

Fig. 11 Centrifugal experimental results from [4] (dashed line) vs. DDA numerical simulation (points).

due in part to neglecting both the additional friction due to air drag and the axial forces in the plane perpendicular to the simulation.

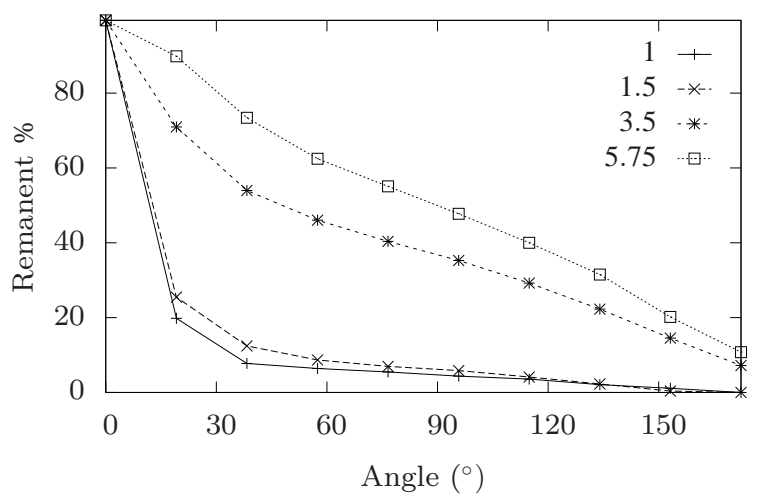

Fig. 12 Simulated remaining material in \% vs. rotation angle for straight bucket and centrifugal discharge for several values of the penalty parameter $\left(K_{N}\right.$ value $=$ number in legend $\times 10^{5}$ $\mathrm{N} / \mathrm{m})$.

Figure 12, the counterpart of Figure 9, shows the percentage of material remaining in the bucket as a function of the rotation angle for several values of $K_{N}$. Since there are many fewer contacts than for gravity discharge, a realistic and smooth process is simulated with smaller penalty parameters $K_{N}>3.5 \cdot 10^{5} \mathrm{~N} / \mathrm{m}$, resulting in a lower computation cost. Due to the high rotational velocity, no recapturing is observed for any $K_{N}$.

\section{Rotational velocity and bucket geometry}

This section studies the evolution of a granular sample under a range of rotational velocities, from low values typical of gravitational discharge to high ones typical of 
centrifugal discharge. Rademacher [14] uses the dimensionless parameter $K_{a}=r \omega^{2} / g$, (usually called in fluid mechanics the Froude number), where $r$ is the variable distance between $c g$ and $c r$. For a concentrated mass, this parameter defines the type of discharge: Gravitational $K_{a}<1$ or centrifugal $K_{a}>1$. The numerical results are compared to the experimental ones from [4]. Again the material for the next two simulations is plastic beads, see section 5 .

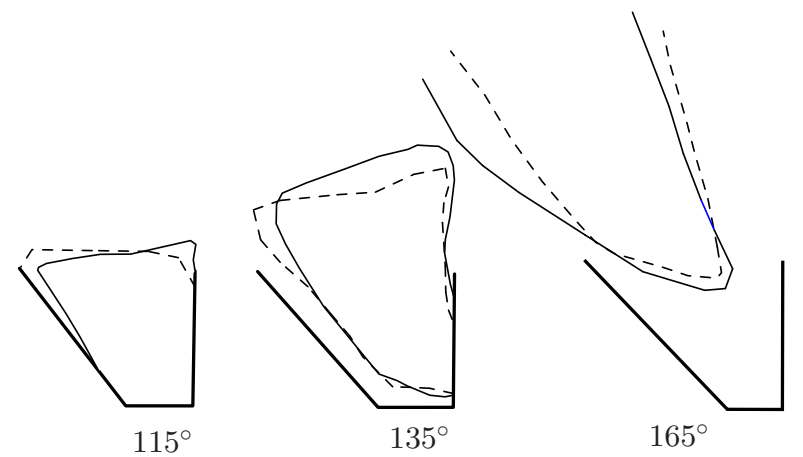

Fig. 13 Experimental (continuous line) and numerical (dashed line) gravitational discharge for several rotation angles. Rademacher number $K_{a}=0.8$.

The simulation, Figure 13, shows that for $K_{a}=0.8$ the discharge begins at $\approx 100^{\circ}$, with the material sliding along the inner wall. When the bucket reaches $135^{\circ}$, the material separates from the outer wall. The effects of the centrifugal force are not observed until the last angles, when the material is ejected from the bucket as a compact volume at an angle of $\approx 165^{\circ}$.

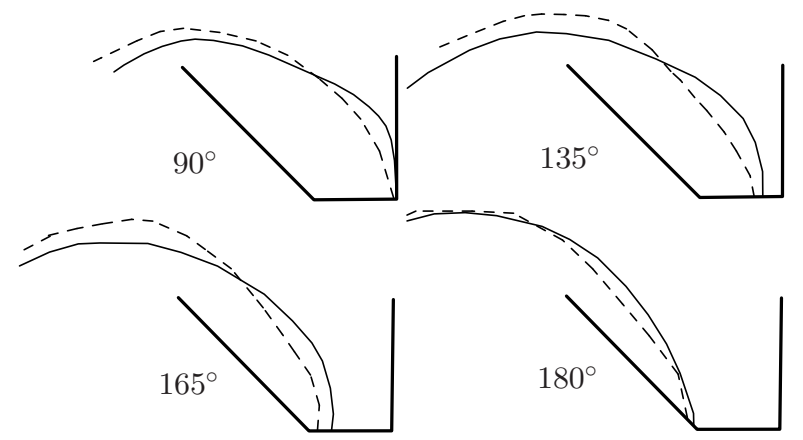

Fig. 14 Experimental (continuous line) and numerical (dashed line) centrifugal discharge for several rotation angles. Rademacher number $K_{a}=2.0$.

When $K_{a}$ is increased to 2.0 (Figure 14), the discharge is predominantly centrifugal, emptying the bucket at a lower rotation angle. The first indications of discharge occur before $90^{\circ}$, with material compactly sliding along the outer wall. Although the bucket is not completely emptied at the end of the rotation, this discharge performs better than the previous one due to the higher velocity.

Figure 15 presents the effect of the bucket opening for the last $180^{\circ}$ rotation angle, comparing the experimental and numerical distributions for centrifugal discharge. The material is now gravel of high density and friction. In the relatively closed bucket $A$ more than $40 \%$ material remains undischarged at the end of the process. As the opening angle increases up to bucket $D$, the percentage is reduced to less than $10 \%$.

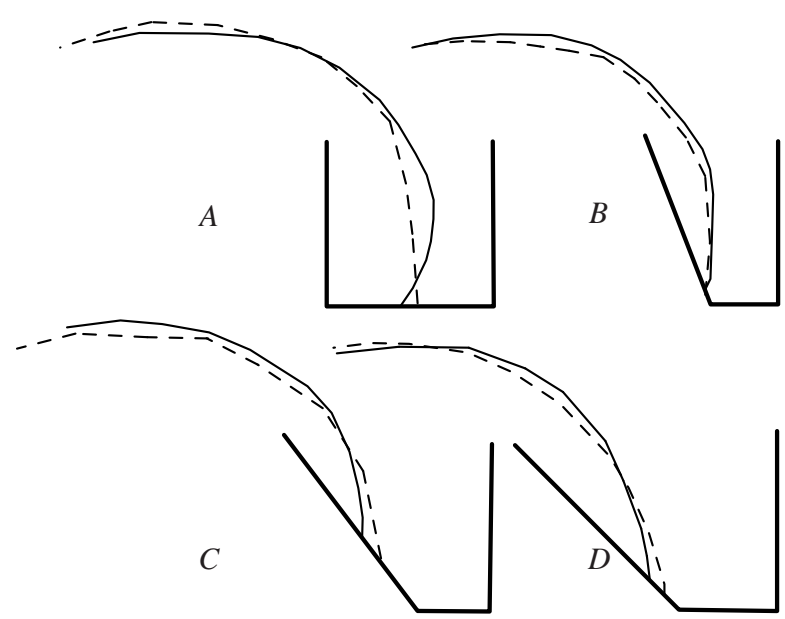

Fig. 15 Influence of bucket openings on remaining material at end of rotation, angle $180^{\circ}$. Experimental (continuous line) and numerical gravitational discharge (dashed line). Rademacher number $K_{a}=2.0$.

Based on these and other cases not presented in the paper, we can draw the following conclusions:

- The rotation angle in which movement of the material begins, $\approx 25^{\circ}$, is constant for any bucket shape, as long as it is initially full.

- When the angle of the bucket outer wall increases, the amount of discharged material also increases.

The last item implies that if the opening angle increases, the rotation angle for initial sliding decreases implying a performance increase.

The quantitative results are given in Table 3, the small differences between the experimental and numerical results are small given the uncertainties in material properties and geometry. From these simulations, we conclude that DDA approximates well the experimental results from [4] for a significant range of velocities and bucket geometries, providing physical support for the numerical results. 
Table 3 Remaining material in \% for last rotation angle $180^{\circ}$ : Experimental, DDA and difference between them.

\begin{tabular}{cccc}
\hline Type & Exp. & DDA & Difference \\
\hline$A$ & 55 & 52 & 3 \\
$B$ & 32 & 29 & 3 \\
$C$ & 19 & 18 & 1 \\
$D$ & 8 & 8 & 0 \\
\hline
\end{tabular}

\section{Optimal design}

\subsection{Parametric analysis}

The present analysis consists on the optimization of the shape of the outer bucket wall for several bulk materials and operational velocities. To the best of our knowledge, the only attempt to optimize a bucket shape for centrifugal discharge is the practical approach from [11], previously presented in [10]. In these two references, the optimal shape is obtained studying the analytical trajectories of a single particle initially located at different positions on the piecewise linearly-discretized outer wall.

In the present work, the optimization is performed using DDA with a large number of particles (up to 4000), taking into account contacts with friction. More realistic results than in [11] are expected thanks to the many interactions and better physics. The criteria to optimize are:

- Maximum horizontal distance reached by the $c g$ of the particle system: cost function $f_{1}$

- Maximum absolute distance traveled by the $c g$ of the particle system: cost function $f_{2}$

- Minimum amount of remaining particles inside the bucket after discharge: cost function $f_{3}$

- Minimum dispersion as measured by the radius of a circle centered at the $c g$ including $80 \%$ of the flying particles: cost function $f_{4}$

All the cost functions are correlated to each other, i.e. $f_{2}$ is directly related with $f_{3}$ since the farther the particles are thrown, the lower the amount of remaining material.

\subsection{Optimization algorithm}

The shape of the outer wall, see Figure 16, is parametrically defined by a hypercubic Bezier curve in the domain $(\mathrm{x}, \mathrm{y}) \in[0,-1] \times[0,1]$ as

$$
\begin{aligned}
(\mathrm{x}, \mathrm{y})= & \theta^{4} x_{1}+4 \theta^{3}(1-\theta) x_{2}+6 \theta^{2}(1-\theta)^{2} x_{3} \\
& +4 \theta(1-\theta)^{3} x_{4}+(1-\theta)^{4} x_{5}
\end{aligned}
$$

with $\theta \in[0,1]$ and where $x_{1}=(0,0)$ and $x_{5}=(-0.4,0.4)$ are the fixed initial and final points. The figure shows a possible concave outer wall, for three arbitrary positions of points $x_{2}, x_{3}$, and $x_{4}$. Fixing the ordinate position of these three points, $x_{2}=\left(\mathrm{x}_{2}, 0.1\right), x_{3}=\left(\mathrm{x}_{3}, 0.2\right), x_{4}=$ $\left(x_{4}, 0.3\right)$, the optimization algorithm aims at determining their abscissae such that the shape of the outer wall produces an optimum discharge. The algorithm chosen is the "gradual deformation optimization algorithm" consisting of the following steps:

1. Generate a group of three abscissae $\left(x_{2}, x_{3}, x_{4}\right)_{i}$ using independent random numbers from the domain $[-1,0] \times[-1,0] \times[-1,0]$.

2. Interpolate the Bezier curve $x_{i}$, simulate discharge and measure the multiobjective cost function $f\left(x_{i}\right)$ :

$$
f\left(x_{i}\right)=\sum_{j=1}^{2} \alpha_{j} \frac{f_{j}\left(x_{i}\right)}{f_{j}\left(x_{0 j}\right)}-\sum_{j=3}^{4} \alpha_{j} \frac{f_{j}\left(x_{i}\right)}{f_{j}\left(x_{0 j}\right)}
$$

3. Generate a second set $\left(\mathrm{x}_{2}, \mathrm{x}_{3}, \mathrm{x}_{4}\right)_{i+1}$ and obtain $x_{i+1}$ and $f\left(x_{i+1}\right)$.

4. Optimize the value of $t \in[0,1]$ in $x_{i+2}=t x_{i}+(1-$ $t) x_{i+1}$ that minimizes $f\left(t x_{i}+(1-t) x_{i+1}\right)$.

5. Assign $x_{i} \leftarrow x_{i+2}$ and go back to item 3 .

In item 2 , the scalars $\alpha_{j},(j=1, \ldots, 4)$ are positive weights assigned to each cost function $f_{j}$. Since each cost function has a different range of possible values, it is necessary to normalize them. To this end, we use the values of the cost function evaluated at an initial vector $x_{0 j}$. Item 4 is a one-parameter optimization that can

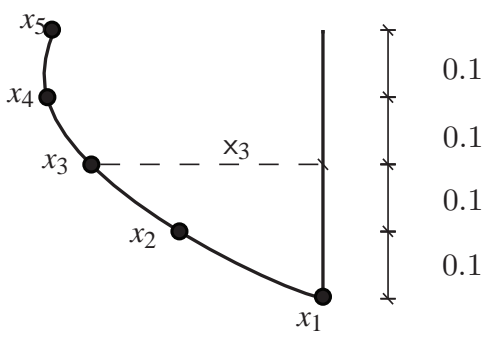

Fig. 16 Knot positions for the definition of optimal bucket outer wall. Dimensions in $m$ and shape defined concave (from bucket inside).

be performed using a standard method such as "golden sections". In this item, the optimal $t_{o p}$ obtained fullfils $f\left(x_{i+2}\right) \leq f\left(x_{i+1}\right)$ and $f\left(x_{i+2}\right) \leq f\left(x_{i}\right)$.

\subsection{Optimal results}

Figure 17 shows the optimized outer wall shapes for two rotational velocities and for several particle diameters, for each optimization criteria considered independently. For centrifugal discharge $C$, the rotation velocity is 10 
$\mathrm{rad} / \mathrm{s}$, and for intermediate discharge $I$, the rotation velocity is $7.85 \mathrm{rad} / \mathrm{s}$. The material is of high density $2200 \mathrm{~kg} / \mathrm{m}^{3}$ and has a high inner friction angle of $40^{\circ}$, corresponding to sand (3 mm diameter, 4000 particles) and to two gravels $(10 \mathrm{~mm}, 300$ particles; and $20 \mathrm{~mm}$, 120 particles). The number of particles is chosen so that they roughly represent the size of real grains.
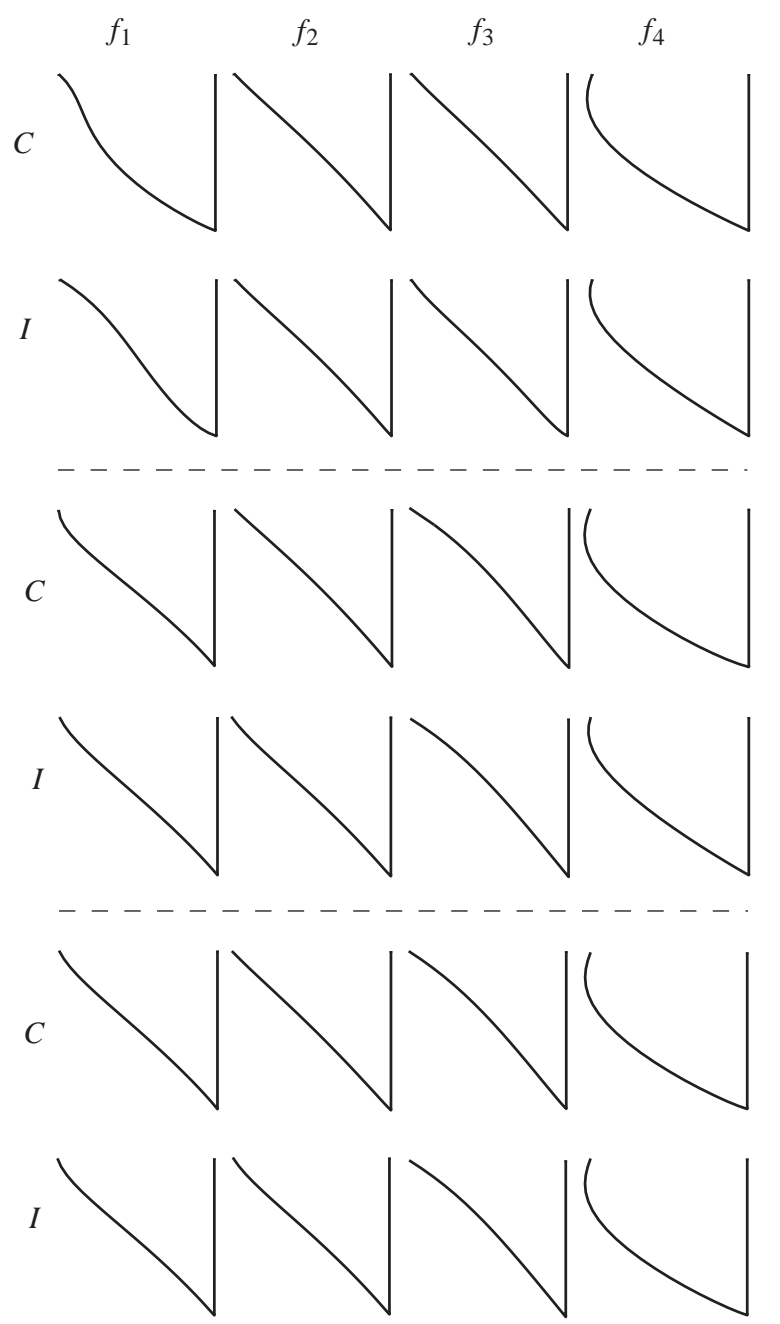

Fig. 17 Optimal external wall when each optimization criteria is considered independently, for heavy and high friction materials with diameter $3 \mathrm{~mm}$ (top), $10 \mathrm{~mm}$ (middle), and $20 \mathrm{~mm}$ (bottom). Centrifugal ( $C$ with $\omega=10.5 \mathrm{rad} / \mathrm{s}$ ) and intermediate discharges ( $I$ with $7.85 \mathrm{rad} / \mathrm{s})$.

For sand, and centrifugal discharge $C$ we obtain an open (T-type or double curvature bucket) or even straight shape when the objective is to discharge as far as possible the material and/or completely empty the bucket $\left(f_{1}, f_{2}, f_{3}\right)$. On the contrary, when the objective is to minimize dispersion $\left(f_{4}\right)$ the result is a closed-shape logarithmic bucket. The intermediate velocity $I$ results are very similar to those of $C$ except for $f_{1}$; the reason is that at lower velocity the bucket needs to be even more open than in $C$ for the material to reach a high horizontal distance. Notice that this small-particle, high frictional sand behaves as a viscous fluid, therefore for both $C$ and $I$ the dispersion outside the bucket is small as long as this sand is compacted during the sliding along the outer wall. Also, due to this fluidity, the tip of the outer wall is curved, outward (convex) for maximum distance or inward (concave) for minimum dispersion. In general, a double curvature favors emptying since during the internal movement the material in contact with the outer wall partially detaches, reducing the friction of the total mass.

For gravel, the predicted outer wall shapes are very similar to those obtained for sand for $f_{2}, f_{3}$ and $f_{4}$ but fairly different for $f_{1}$. This is so for both particle diameters and velocities: The interstitial space among particles or voids is now relevant and the number of contacts (consequently friction) smaller than before; in addition, the momentum of each individual particle at ejection is larger due to the larger individual mass. Therefore, not much curvature is needed at ejection. Functions $f_{1}$ (maximum horizontal distance) and $f_{3}$ (minimum undischarged material) demand opposite shapes at ejection; this is the reason for which the outside curvature changes sign. At intermediate velocities the discharge is not completely by sliding along the other wall but rather a compact "take off", see Figure 13. Therefore, to prevent premature discharge, the optimized outer wall intends to "attract" the particles. Gravels, as opposed to sand, do not behave as a fluid, and include a substantial number of voids, what results in very similar shapes for almost all objectives, for both gravels and for the two velocities analyzed.

The optimal values of the objective functions are shown in Table 4: Distances related to $f_{1}$ and $f_{2}$ almost double between sand and gravel; with a small difference between the two gravel diameters. As expected, the minimum remaining material $f_{3}$ is almost zero for $C$ but significant for $I$. Finally, the dispersion outside the bucket $f_{4}$ is small for sand and larger for gravels. This dispersion in the gravels is due to the mentioned higher momentum and to the existence of less contacts, and therefore less energy dissipation, with respect to sand.

New simulations were done for discharges of smelting slag, a material with very low density, $1200 \mathrm{~kg} / \mathrm{m}^{3}$ and also low internal friction angle, $12^{\circ}$; Figure 18 shows the only shape that is different from those of Figure 17. This shape corresponds to the maximum horizontal distance for centrifugal discharge of $3 \mathrm{~mm}$ diameter sand. The discrepancy of this single case is due to the low friction and low density; the material easily escapes the 
Table 4 Quantified results for the optimizing functions in centrifugal, intermediate discharges and three particle diameters. Outer wall shapes given in Figure 17.

\begin{tabular}{cccccc}
\hline Discharge & Size & $f_{1}$ & $f_{2}$ & $f_{3}$ & $f_{4}$ \\
\hline & $\mathrm{mm}$ & $\mathrm{m}$ & $\mathrm{m}$ & $\%$ & $\mathrm{~m}$ \\
\hline \multirow{2}{*}{$C$} & 3 & 0.78 & 0.84 & 0.0 & $\mathbf{0 . 2 0}$ \\
& 10 & 1.53 & 1.82 & 1.0 & 0.63 \\
& 20 & 1.64 & 1.92 & 0.0 & 0.61 \\
\hline \multirow{2}{*}{$I$} & 3 & 0.66 & 0.84 & 6.0 & 0.18 \\
& 10 & 1.32 & 1.58 & 4.0 & 0.52 \\
& 20 & 1.47 & 1.62 & 2.0 & 0.54 \\
\hline
\end{tabular}

bucket resulting in a discharge almost equal to that of I for the heavy material of Figure 17, left second row.

About the numerical results for smelting slag (not shown), it can be mentioned that the distances and the dispersion are much higher than for high density material, and that the remanent material is zero. Again, this is due to the lower friction and lower energy acquired by the light material during bucket rotation.

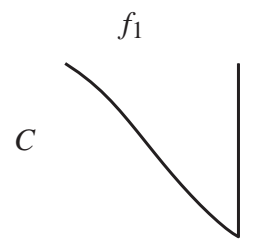

Fig. 18 Optimal external wall for light smelting slag material; results are almost equal to those in Figure 17 except for the one shown corresponding to centrifugal discharge at 10.5 $\mathrm{rad} / \mathrm{s}$, maximum distance and sand of $3 \mathrm{~mm}$ diameter.

Finally, Figure 19 shows the evolution of the bucket shape for $3 \mathrm{~mm}$ diameter sand and centrifugal discharge when the four criteria are combined into a multiobjective optimization.

In the top row, $\alpha_{1}=\alpha_{2}=\alpha_{3}=1$, while $\alpha_{4}$ takes the values $1,2,4,6,8$, from left to right. For the lowest values of $\alpha_{4}$, the optimization gives a T-type (see Figures 3 ), since the added contribution of $f_{1}, f_{2}$ and $f_{3}$ is dominant over that of $f_{4}$ and these three objectives require approximately similar shapes (except at the outer wall end). Comparing these shapes to those from the Figure 17 we can notice that the optimal shape combines the lower zone of the wall from the optimal $f_{1}$, and the upper zone from the optimal $f_{2}$ or $f_{3}$ but with more curvature. As the weight of $f_{4}$ increases twofold for each figure, intermediate combinations of T-type and logarithmic shapes result, until a pure logarithmic one is given for $\alpha_{4}=8$ at the top right of Figure 17. The optimal shape for $\alpha_{4}=4$ in the center shows a strong double curvature intended to minimize dispersion and reach maximum distance at the same time: The bottom curve enforces the concentration of particles to minimize dispersion; the compacted particles travel along the wall until the curvature changes, then they lose contact and take off. For the optimal shape for $\alpha_{4}=2$, center-left, the double curvature is almost unnoticeable and the curvature change is close to the wall bottom; therefore, this shape almost exclusively favors the detachment of the concentrated particles. Finally, the optimal shape for $\alpha_{4}=6$, center-right, is a transition from the unusual center figure and the one in the right when the weight $\alpha_{4}=8$, any convexity disappears and the shape is fully logarithmic, as in current practical buckets.

Table 5 Evolution of horizontal and total distances, remaining material and dispersion values when the weight of the objective function minimizing dispersion is increased.

\begin{tabular}{ccccc}
\hline$\alpha_{j}$ & $f_{1}$ & $f_{2}$ & $f_{3}$ & $f_{4}$ \\
\hline & $\mathrm{m}$ & $\mathrm{m}$ & $\%$ & $\mathrm{~m}$ \\
\hline $1 / 1 / 1 / 1$ & 0.76 & 0.79 & 0.5 & 0.54 \\
$1 / 1 / 1 / 2$ & 0.74 & 0.77 & 2.0 & 0.48 \\
$1 / 1 / 1 / 4$ & 0.58 & 0.63 & 5.3 & 0.33 \\
$1 / 1 / 1 / 6$ & 0.52 & 0.57 & 6.7 & 0.27 \\
$1 / 1 / 1 / 8$ & 0.43 & 0.48 & 15 & $\mathbf{0 . 2 0}$ \\
\hline
\end{tabular}

Table 5 contains the optimal values of the objective functions for the shapes of the top row in Figure 19. The first row is for equal weights, with results similar to those in the first row in Table 4, corresponding to the top left bucket of Figure 17. The only significantly difference value is for $f_{4}$, since as mentioned before, with this combination of weights, the first three functions are predominant. As $\alpha_{4}$ increases while the other weights are kept constant, the bucket performance decreases with respect to $f_{1}, f_{2}$ and $f_{3}$; for a high $\alpha_{4}=8$ we recover the optimal dispersion radius of $\mathbf{0 . 2 0} \mathrm{m}$ with numbers typical of the logarithm bucket: modest travel distances (only $43 \%$ of the optimal) and with a significant remaining material of $15 \%$.

In the bottom row of Figure $19 \alpha_{1}=\alpha_{3}=\alpha_{4}=1$, while $\alpha_{2}$ takes the values $1,2,4,6,8$, from left to right. Starting with the same optimal shape obtained in the top row, the twofold increment of $\alpha_{2}$ does not induce as significative changes as when $\alpha_{4}$ is the variable weight. The only noticeable change is the curvature of the bottom part of the bucket that goes from slightly concave to straight to slightly convex as $\alpha_{2}$ increases.

The optimization of the gravity discharge is much simpler than the previous ones and is not shown here: This discharge occurs exclusively along the inner wall and the only relevant parameter is the deflector inclination, and to a lesser extend, its length. 


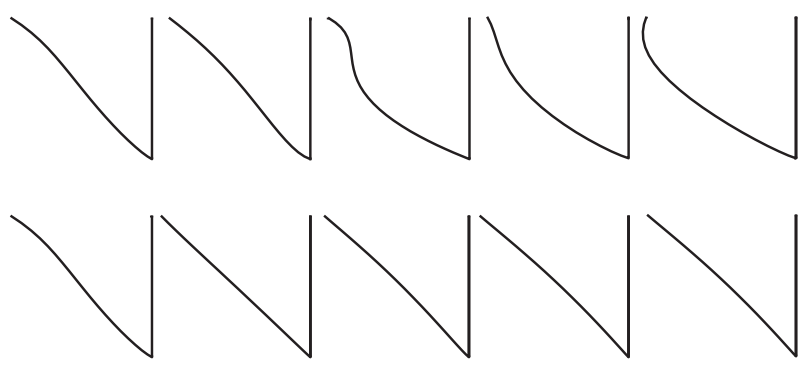

Fig. 19 Outer wall shape evolution when all objective functions are considered. Sensitivity to the weight applied to objective function $f_{4}$ (top row) and to objective function $f_{2}$ (bottom row). Centrifugal discharge, high density and friction sand of $3 \mathrm{~mm}$ diameter.

\section{Conclusions}

The Discrete Deformation Analysis (DDA) method has been used to analyze the bucket elevator discharge of granular materials for several velocities, bulk materials and bucket shapes. After a brief theoretical introduction on solid rigid mechanics of contact and friction, numerical results have been compared with analytical and experimental ones obtaining a good agreement. The parametrical analyses have allowed us to explain the most significant aspects of the behavior of granular materials inside and outside of the most common bucket types. In addition, these analyses have permitted to define the most influential discharge parameters for each bucket type.

Traditionally, the optimization of the bucket shape has been done using experimental or simple analytical procedures based in a single particle, but DDA can be used as an important part of this optimization process. Cost functions have been defined to maximize the reached horizontal and absolute distances, and to minimize the material left in the bucket and the dispersion after exiting. With these functions and using the gradual deformation optimization algorithm, bucket shapes have been automatically designed for heavy frictional material (sand, gravel) and light low-frictional slag.

Practical conclusions can be drawn from these analyses. For instance, the optimal shapes change depending which is the main objective chosen; combining the different objectives the optimal shapes result in hybrid combinations of the optimal shapes for each objective. It is demonstrated that the classical T- and logarithmic types are optimal for opposite objectives in the discharge, and that intermediate shapes can achieve compromise results. The analyses presented in this work are therefore considered a useful tool to design the most efficient shape of the bucket for any specific working conditions.
Acknowledgements J.L. Pérez-Aparicio, R. Bravo were partially supported by the MFOM I+D (2004/38), both by MICIIN \#BIA 2008-00522 and the first also by Polytechnic University of Valencia under grant PAID 05-10-2674. J.J. GómezHernández was partially supported by MICIIN \#CGL 201123295.

\section{References}

1. Balevičius, R., Kačianauskas, R., Mroz, Z., Sielamowicz, I.: Discrete element method applied to multiobjective optimization of discharge flow parameters in hoppers. Structural and Multidisciplinary Optimization 31(3), 163-175 (2006)

2. Beckert, R., Föll, R.: Untersuchung der abwurfverhältnisse an kettenbecherwerken. Förden Heben 1(15), 833836 (1966)

3. Belytschko, T., Liu, W., Moran, B.: Nonlinear Finite Elements for Continua and Structures. Wiley (2000)

4. Beverley, G.: Mechanics of high speed bucket elevator discharge. Ph.D. thesis, University of Newcastle (1986)

5. Beverley, G., Roberts, A., Hayes, J.: Mechanics of high speed elevator discharge. Bulk Solids Handling 3(4), 853859 (1983)

6. Bravo, R., Pérez-Aparicio, J., Laursen, T.: An enhanced energy conserving time stepping algorithm for frictionless particle contacts. International Journal for Numerical Methods in Engineering 85(11), 1415-1435 (2011)

7. Bravo, R., Pérez-Aparicio, J., Laursen, T.: An energy consistent frictional dissipating algorithm for particle contact problems. International Journal for Numerical Methods in Engineering 92(9), 753-781 (2012)

8. Gao, Y., Muzzio, F., Ierapetritou, M.: Optimizing continuous powder mixing processes using periodic section modeling. Chemical Engineering Science 80, 70-80 (2012)

9. Hu, L.: Gradual deformation and iterative calibration of gaussian-related stochastic models. Mathematical Geology 32(1), 87-108 (2000)

10. Jaskulski, A.: Methodology of multi-criteria optimization of appliances for vertical grain transportation. Ph.D. thesis, Warsaw University of Technology (1992)

11. Jaskulski, A.: Engineer-to-order approach to high speed bucket elevator design in a small-enterprise. Applied Engineering in Agriculture 24(5), 545-557 (2008)

12. Korzen, Z.: Mechanics of gravitational discharge of cellless bucket wheels in reclaiming machines. Bulk Solids Handling 7(6), 801-812 (1987)

13. Korzen, Z., Dudek, K.: Mathematical model of the operational efficiency of a multibucket centrifugal discharge wheel [model matematyczny wydajnosci roboczego procesu kola wieloczerpakowego z odsrodkowym wysypem]. Politechnika Warszawska, Prace Naukowe, Mechanika 1(121), 187-199 (1989)

14. Korzen, Z., Dudek, K.: Reclaiming with a high-speed bucket wheel with centrifugal discharge. Bulk Solids Handling 11(3), 615-626 (1991)

15. Koster, K.: Use of high-capacity bucket elevators in the cement industry. [zum einsatz von hochleistungsbecherwerken in der zementindustrie]. Zement-Kalk-Gips 33(3), 116-119 (1980)

16. Koster, K.: Bulk material discharge of bucket elevators, especially high-capacity bucket elevators. [zum schuettgutabwurf bei becherwerken, insbesondere bei hochleistungsbecherwerken]. Aufbereitungs-Technik 25(8), 450-463 (1984) 
17. Koster, K.: Centrifugal discharge of bucket elevators. Bulk Solids Handling 5(2), 449-460 (1985)

18. Koster, K.: Problem of complete emptying of high-speed elevator buckets. Aufbereitungs-Technik 27(9), 471-481 (1986)

19. Koster, K.: Development and state of the art in heavyduty bucket elevators with central chains - part 2 [entwicklung und stand der technik von hochleistungsbecherwerken mit zentralkette - teil 2]. ZKG International 49(4), 173-187 (1996)

20. Kruggel-Emden, H., Sudbrock, F., Wirtz, S., Scherer, V.: Experimental and numerical investigation of the bulk behavior of wood pellets on a model type grate. Granular Matter 14(6), 681-693 (2012)

21. Makokha, A., Moys, M., Bwalya, M., Kimera, K.: A new approach to optimising the life and performance of worn liners in ball mills: Experimental study and dem simulation. International Journal of Mineral Processing 84(14), 221-227 (2007)

22. McBride, W., Sinnott, M., Cleary, P.: Discrete element modelling of a bucket elevator head pulley transition zone. Granular Matter 13(2), 169-174 (2011)

23. Moon, T., Oh, J.: A study of optimal rock-cutting conditions for hard rock tbm using the discrete element method. Rock Mechanics and Rock Engineering 45(5), 837-849 (2012)

24. Moosavi, M., Grayeli, R.: A model for cable bolt-rock mass interaction: Integration with discontinuous deformation analysis (dda) algorithm. International Journal of Rock Mechanics and Mining Sciences 43(4), 661-670 (2006)

25. Pérez-Aparicio, J., Bravo, R.: Discrete Elements, vol. 2, pp. 41-77. Consorcio TCN (2006)

26. Pérez-Aparicio, J., Bravo, R., Ortiz, P.: Refined element discontinuous numerical analysis of dry-contact masonry arches. Engineering Structures 48, 578-587 (2013)

27. Rademacher, F.: Non-spill discharge characteristics of bucket elevators. Powder Technology 22(2), 215-241 (1979)

28. Shi, G.: Discontinuous deformation analysis: A new model for the statics and dynamics of block systems. Ph.D. thesis, University of California, Berkeley (1988)

29. Shi, G., Goodman, R.: Two dimensional discontinuous deformation analysis. International Journal for Numerical and Analytical Methods in Geomechanics 9(6), 541556 (1985)

30. Shmulevich, I.: State of the art modeling of soil-tillage interaction using discrete element method. Soil and Tillage Research 111(1), 41-53 (2010)

31. Walton, O., Moor, C., Gill, K.: Effects of gravity on cohesive behavior of fine powders: implications for processing lunar regolith. Granular Matter 9(5), 353-363 (2007) 